\title{
BMJ Open Methods for estimating economic benefits of surgical interventions in low- income and middle-income countries: a scoping review
}

\author{
Amy Hilla, ${ }^{1}$ Victoria Reese, ${ }^{2}$ Justice Nonvignon, ${ }^{3}$ Carrie B. Dolan (D) ${ }^{4}$
}

To cite: Hilla A, Reese $\mathrm{V}$, Nonvignon J, et al. Methods for estimating economic benefits of surgical interventions in low-income and middle-income countries: a scoping review. BMJ Open 2020;10:e039644. doi:10.1136/ bmjopen-2020-039644

- Prepublication history and additional material for this paper is available online. To view these files, please visit the journal online (http://dx.doi.org/10. 1136/bmjopen-2020-039644).

Received 22 April 2020 Revised 31 October 2020 Accepted 02 November 2020

Check for updates

(C) Author(s) (or their employer(s)) 2020. Re-use permitted under CC BY-NC. No commercial re-use. See rights and permissions. Published by BMJ.

${ }^{1}$ Economics, William \& Mary, Williamsburg, Virginia, USA

${ }^{2}$ Applied Math and Economics, William \& Mary, Williamsburg, Virginia, USA

${ }^{3}$ Health Policy, Planning, and Management, University of

Ghana, Legon, Ghana

${ }^{4}$ Health Sciences, William \&

Mary, Williamsburg, Virginia, USA

Correspondence to

Dr Carrie B. Dolan;

cbdolan@wm.edu

\section{ABSTRACT}

Objectives Studies indicate that many types of surgical care are cost-effective compared with other health interventions in low-income and middle-income countries (LMICs). However, global health investments to support these interventions remain limited. This study undertakes a scoping review of research on the economic impact of surgical interventions in LMICs to determine the methodologies used in measuring economic benefits. Design The Arksey and 0'Malley methodological framework for scoping reviews and the Preferred Reporting Items for Systematic Reviews and MetaAnalyses Extension for Scoping Reviews checklist were used to review the data systematically. Online databases were used to identify papers published between 2005 and 2020, from which we selected 19 publications that quantitatively examined the economic benefits of surgical interventions in LMICs.

Results Majority of publications (79\%) reported the use of disability-adjusted life-years (DALYs) to assess economic impact. In comparison, $21 \%$ used other measures, such as the value of statistical life or cost-effectiveness ratios, or no measure at all. $31 \%$ were systematic or retrospective reviews of the literature on surgical procedures in LMICs, while $69 \%$ either directly assessed economic impact in a specific area or evaluated the need for surgical procedures in LMICs. All studies reviewed related to the economic impact of surgical procedures in LMICs, with most about paediatric surgical procedures or a specific surgical specialty.

Conclusion To make informed policy decisions regarding global health investments, the economic impact must be accurately measured. Researchers employ a range of techniques to quantify the economic benefit of surgeries in LMICs, which limits understanding of overall economic value. We conclude that the literature would benefit from a careful selection of methods, incorporating age and disability weights based on the Global Burden of Disease weights, and converting DALYs to dollars using the value of statistical life approach and the human capital approach, reporting both estimates.

\section{INTRODUCTION}

The need for more surgical interventions in low-income and middle-income countries (LMICs) has been widely advocated. ${ }^{12}$ Each
Strengths and limitations of this study

- This review is organised according to the Arksey and 0'Malley framework and the Preferred Reporting Items for Systematic Reviews and Meta-Analyses extension for Scoping Reviews (PRISMA-ScR) checklist () to map the methodological approaches used to examine the economic impact of surgical interventions in low-income and middle-income countries (LMICs).

- This scoping review highlights the importance of careful consideration of the quality of data, calculation of disability-adjusted life-years and the applications of weights when examining the economic efficiency of surgical interventions in LMICs.

- This review includes an overview of a range of study designs and methodologies, but it was not designed to critically appraise or synthesise evidence as typical in a systematic review.

- This scoping review is useful to researchers and policy-makers to reduce duplication of effort and guide future research questions that aim to measure the economic impact of surgical interventions in LMICs.

year, there are approximately 143 million additional surgical procedures needed in LMICs, and over 5 billion people cannot access safe and affordable surgical care and anaesthesia. ${ }^{3}$ Continued failure to invest in surgical interventions before 2030 could result in an estimated US\$12-US\$13 trillion loss in economic productivity. ${ }^{3}$ Thus, the hypothesis that surgical interventions and economic productivity are related deserves attention from both researchers and policy-makers.

Recent studies have indicated that many areas of surgical care are cost-effective compared with other common health interventions. ${ }^{4-6}$ Investing in these services is affordable, saves lives, and promotes economic growth by strengthening the entire health system. ${ }^{13}$ Up to $2 \%$ of economic growth is lost due to untreated surgical conditions in 
LMICs. ${ }^{3}$ Studies show that there are multiple areas within procedure groups, including inguinal hernia repair, trichiasis surgery, cleft lip and palate repair, circumcision, congenital heart surgery and orthopaedic procedures that offer quantifiable economic value. ${ }^{7}$ Other studies demonstrated that significant economic benefits are lost when there are long waiting times for surgical procedures in LMICs. ${ }^{8}$ The literature supports both macro and microlevel economic improvements resulting from surgical interventions, suggesting that increasing surgical capacity should be a global health priority. ${ }^{9}$ However, the scale up of global health investments to support these interventions remains limited. ${ }^{1}$

Studies have measured the economic impact of surgical interventions in LMICs. However, the studies were constrained by scarce and limited data, limitations in disability weights, and significant gaps in research methods. $^{2}{ }^{9} 10$ Standardisation of economic analysis methods in other areas of global health can be identified but do not exist when looking at similar research on surgical procedures. A wide range in the quality of studies contributes to a limited commitment by global health stakeholders to provide better global surgical care. Although previous systematic reviews have been valuable in adding to the literature describing the economic impact of surgical interventions, they were not designed to provide a broad overview of the methodological approaches on the economic impact of surgical interventions in LMICs. Instead, the previous reviews were designed to carefully summarise the best available information on a specific research question related to children's surgical care and disability weights. This approach may have missed studies that employed a range of designs and methods. To compensate for this methodological gap, we undertook a scoping review to map the existing literature specific to the methodological approaches used to examine surgical interventions' economic impact in LMICs. It summarises 'industry-standard' methods and what methods prior researchers have used to estimate economic impact. This scoping review is useful to researchers and policy-makers to reduce duplication of effort and guide future research questions that aim to measure the economic impact of surgical interventions in LMICs. It is also valuable to organisations that coordinate short-term medical missions in LMICs that may want to evaluate their own programmes' effectiveness.

\section{METHODS}

Both scoping and systematic reviews require a robust and structured search of the literature. Unlike systematic reviews, scoping reviews do not formally assess the quality of studies and synthesise conclusions related to a specific question. Scoping reviews are particularly useful in systematically mapping key concepts and research gaps in a body of literature. ${ }^{11}$ The purpose of this scoping review was to identify the methodological approaches used to measure economic impact. This study described the five stages of a scoping review described by Arksey and O'Malley, and the Preferred Reporting Items for Systematic Reviews and Meta-Analyses extension for Scoping Reviews checklist were used to review the data systematically. ${ }^{12} 13$

\section{Stage 1: identifying the research question}

Arksey and O'Malley emphasise the importance of carefully considering the implications of adopting specific positions while developing the research question. ${ }^{12}$ Through an iterative process, the research question was refined from the narrow perspective of understanding the economic benefits to a wide approach to generate broad coverage in this scarce literature. The overall research question of this scoping review is: 'What methods have been implemented to examine the economic benefits of surgical interventions in LMICs?' For this review, methods are defined as quantitative approaches implemented to measure economic benefits. In the context of this work, we adopted a broad definition of economic benefit. We included articles that used standard economic evaluations metrics such as disability-adjusted life-years (DALYs) and life-years saved.$^{5}$

\section{Stage 2: identifying relevant studies}

We searched multidisciplinary electronic databases of the published literature, including Academic Search Complete, PubMed, ScienceDirect, EconLit, Google Scholar and LILACs. For practical purposes, the search was limited to studies published in English. The search strategy was collaboratively and iteratively developed with the research team and research collaborators. The search strategy used keywords related to economic impact evaluation surgery, economic evaluation surgery LMICs, surgical economic outcomes, paediatric surgery's economic impact and measuring surgical outcomes. We specifically included paediatric surgery search terms because accurate measurement of the burden of children's paediatric surgery is critical, and accurate measurement has primarily relied on estimates from modelling studies instead of primary data collection. ${ }^{10}$ Consistent keywords were used across all six electronic databases. Keywords were established and chosen to identify any research potentially related to the economic benefits of surgery in LMICs, or any parallels, derivations, or specialties of those topics. The search strategy was initially implemented in September 2019 and was repeated in December 2019 and July 2020, before submission. After searching, 33 articles were identified as potentially relevant and evaluated, and 19 were included in the review (figure 1). Additional details on the search strategy are outlined in online supplemental table 1. Summaries of each article review are available in online supplemental table 2 .

\section{Stage 3: study selection}

The eligibility criteria for articles included in the review met the following criteria: 


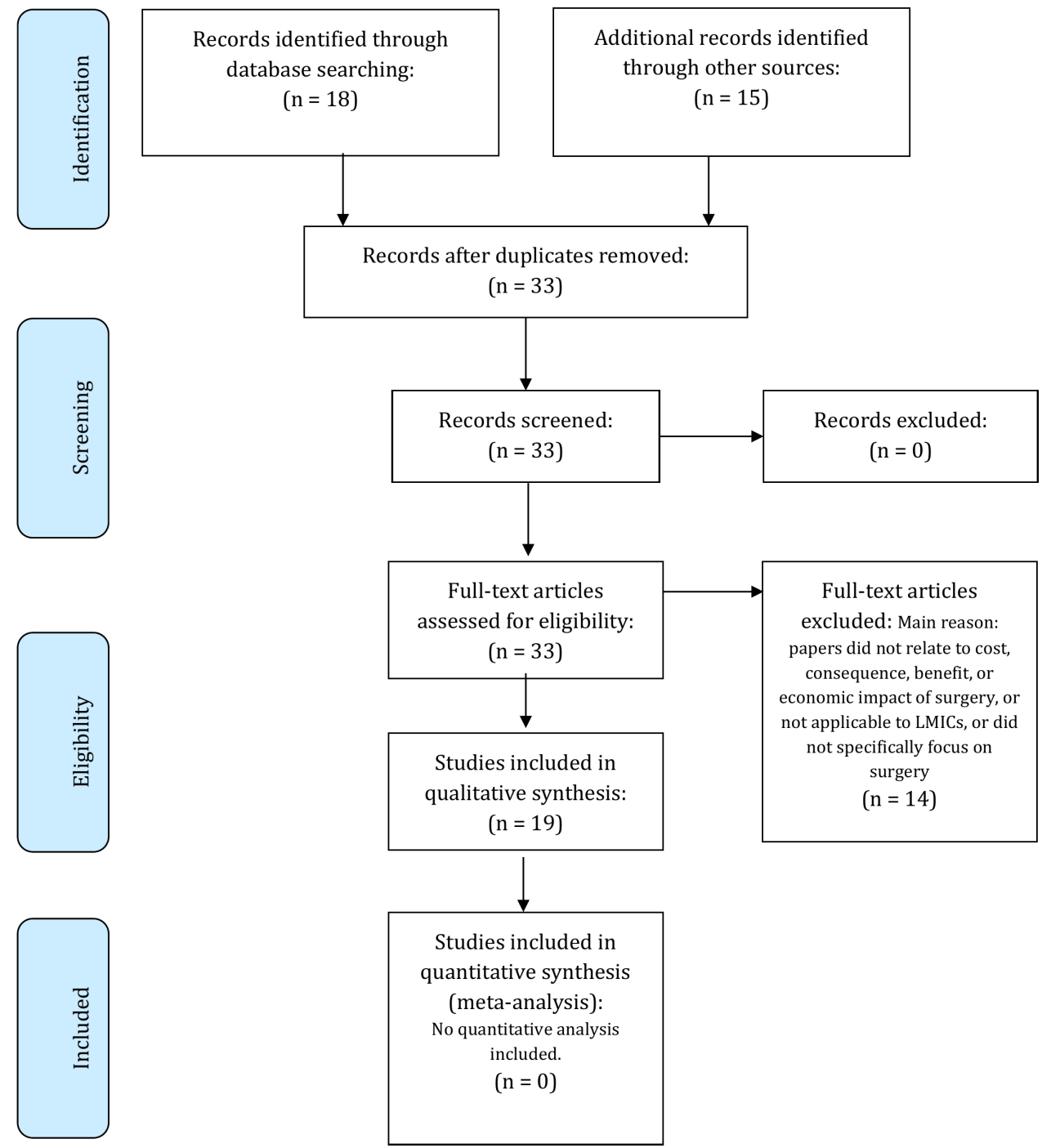

Figure 1 PRISMA-ScR diagram of screening protocol. LMIC, low-income and middle-income country; PRISMA-ScR, preferred reporting Items for systematic reviews and meta-analyses extension for scoping reviews.

\section{Inclusion criteria}

- Study setting: LMICs.

- Intervention: surgical procedures.

- Study design: quantitative designs measuring the economic benefits of surgery (cost-effectiveness, costutility, cost-benefit).

- Time: studies published after 2005.

- Research articles and review articles including systematic reviews.

\section{Exclusion criteria}

- Studies that focused on the economic benefits of general medical procedures (not surgical) were excluded.

- Studies published in a language other than English were excluded.

This scoping review outlined the quantitative methods used to examine surgical interventions' economic benefits in LMICs. Therefore, the reviewers emphasised the methodological section during the search and review of each article. Articles were considered for inclusion only if the methodology section was clear, precise, and concise. This was defined as including: (1) a definition of both cost and consequences, (2) a description of inclusion and exclusion criteria, and (3) a description of the analytic framework.

\section{Stage 4: charting the data}

Two reviewers (AH and VR) independently extracted data from the studies included in the review. The characteristics extracted included: author, year, journal, study title, data source, empirical method, surgical method, measurement of cost and benefit and a summary. This approach was piloted on four studies in order to ensure the extraction was consistent with the research question. To ensure accurate data collection, the reviewers reviewed and compared the abstractions for consistency. The data was compiled into an excel spreadsheet for validation and coding.

\section{Stage 5: data summary and synthesis}

The fifth and final stage of Arksey and O'Malley's scoping review framework summarises and reports findings which are presented in the subsequent section. ${ }^{12}$ 


\section{PATIENT AND PUBLIC INVOLVEMENT STATEMENT}

Patient-level data were not involved in this research.

\section{FINDINGS}

This scoping review yielded 19 articles. In this section, we present articles that focus on our initial research question: What methods are used to assess the economic impact of surgical interventions in LMICs? Each study was reviewed for two criteria: (1) analytical approaches used to measure the economic impact of surgery in LMICs and (2) limitations in analytical approaches used to measure the economic impact of surgical interventions in LMICs.

\section{How are researchers measuring the potential economic benefits of surgical interventions in LMICs?}

Concerning analytical techniques, cost-effectiveness analysis was the most common technique employed. Within this technique, two approaches were identified to quantify the dollar value of DALYs averted: (1) human capital and (2) value of statistical life (VSL). Generally, these approaches produced individual estimates for different subspecialties of surgery. In addition to providing procedure-level estimates, this approach also gives researchers a baseline formula that can subsequently incorporate additional weights. ${ }^{16-814-20}$

In the human capital approach, the total number of DALYs are multiplied by the country's gross national income to estimate the dollars gained by eliminating the disease. ${ }^{21}$ Although the human capital approach provides an estimate of the individual contribution to the national economy, it generally provides lower estimates because it does not account for other unobservable contributions an individual may make to society throughout the lifetime. ${ }^{15}$

The second approach was to calculate the value of a statistical life that estimates an individual's worth based on their value. This amount was calculated by comparing the incomes of relatively risky vs relatively safe jobs. Estimates from the VSL approach tend to be much larger than the human capital approach because individuals tend to value their lives beyond their income. Due to this discrepancy, some authors used both the human capital and VSL approaches and reported both estimates. ${ }^{140}$

In addition to measuring economic benefits through economic evaluations, researchers used other approaches to quantify surgical interventions' economic impact. Raj $e t a t^{2}$ measured the relationship between Risk Adjustment Congenital Heart Surgery, hospital stays, intensive care unit stays and socioeconomic status with the family cost of congenital heart surgery using an ordinary least squares linear regression model, adjusting for potential confounding variables. Other work used a value of lost output approach based on the WHO's Projecting the Economic Cost of Ill-Health model to model how the disease affects the labour force and capital stock. ${ }^{23}$

What are the limitations in analytical approaches used to measure the economic impact of surgical interventions in LMICs?

Articles in the scoping review acknowledge that the available data to measure surgical interventions' economic relationship is limited. ${ }^{39}$ Estimates suffer from limited or omitted cost data on utilisation, depreciation and overhead. ${ }^{2}$ Second, there is an inconsistency in the application of disability weights. ${ }^{10} 24$ Smith $e t a l^{10}$ found limitations in how disability weights for surgical procedures were assigned across studies due to a lack of granularity, wide variability in estimates and lack of inclusion of surgically significant variables such as disease severity and treatment efficacy. One review also found that disability weights were most frequently calculated using the Global Burden of Disease studies data. However, only $18 \%$ accounted for disease severity, and $18 \%$ accounted for postoperative disability. Finally, within the reviewed literature, researchers calculated DALYs multiple ways. Thirteen articles calculated DALYs as the years of life lost to a disease plus the years of life lived with disability. Since some types of surgical interventions, such as reconstructive surgery, do not directly result in years of life lost, one researcher estimated DALYs equal to years of life lived with disability. Saxton $e t a l^{2}$ highlight these issues, recommending that further research solidify how DALYs and disability weights are used to evaluate economic impact, accounting for the issues caused by surgical procedures' complexity and the difficulty of identifying surgical components of multidisciplinary care. Shrime $e t a l^{9}$ likewise notes the wide variation in how DALYs are calculated and includes an equation to obtain an accurate estimate of DALYs averted, emphasising the importance of including disability weight, risk of death, probability of successful treatment and risk of permanent disability estimates in the calculation.

Across all of the papers reviewed, it is clear that DALYs are a useful way to assess cost-effectiveness. Given the wide variety of models, we believe that future research might benefit from allowing variation within cost-effectiveness models. For instance, different disability weights depending on what diseases are being examined, variables to account for differences in Gross National Income across countries, or variables like the probability of future disability are all methods that allow DALY estimates to be more precise. Also, to strengthen surgical systems, the literature needs to study the argument as to whether and how much economic loss occurs due to failure to provide surgical care. The avoidable mortality and morbidity resulting from poor and absent surgical systems leads to economic loss at the household, local, national, regional and global levels but is not thoroughly explored in the existing literature.

\section{DISCUSSION}

It is evident from the current review that there are various approaches used to measure the economic impact of surgical interventions in LMICs. Although the most common approach discerned from our scoping review was an economic impact evaluation using a human capital or VSL approach, the economic impact has also been modelled using regression frameworks. The literature is limited by data quality, discrepancies in the calculation of DALYs and inconsistent application of weights. Future research needs to consider how each of these factors is incorporated into a methodological framework when considering a more specific research 
question of interest. This review has strengths and limitations. The comprehensive literature search's primary strengths are the explicit focus on the methodology, which previous reviews have not systematically reviewed. This review includes an overview of a range of study designs and methodologies, but it was not designed to critically appraise or synthesise evidence as typical in a systematic review. Additionally, since this review was not designed to assess the quality of included studies, it cannot be used to make specific policy recommendations. However, it can be used to identify research questions and inform the development of research strategies. Surgical interventions save lives and promote economic growth. ${ }^{3}$ There is a need for more robust evidence to begin the process of empirically testing this association. The studies reviewed in this article demonstrate the importance of careful consideration of the quality of data, calculation of DALYs, and careful application of weights when examining surgical interventions' economic efficiency in LMICs.

Acknowledgements Helpful and timely comments were provided by Charles Coleman, Henry Rice, Caris Grimes and Jaymie Henry.

Contributors AH: Conceptualisation, data curation, writing-original draft preparation. VR: Conceptualisation, data curation, writing-original draft preparation. $\mathrm{JN}$ : Conceptualisation, writing-review and editing. CD: Methodology, project administration, supervision, writing original draft preparation, writing-review and editing, resources.

Funding This work was funded through a generous grant from the World Pediatric Project 790621.

Disclaimer The funder had no role in study design, data collection, analysis, decision to publish, or manuscript preparation.

Competing interests None declared.

Patient consent for publication Not required.

Provenance and peer review Not commissioned; externally peer reviewed.

Data availability statement № additional data are available.

Supplemental material This content has been supplied by the author(s). It has not been vetted by BMJ Publishing Group Limited (BMJ) and may not have been peer-reviewed. Any opinions or recommendations discussed are solely those of the author(s) and are not endorsed by BMJ. BMJ disclaims all liability and responsibility arising from any reliance placed on the content. Where the content includes any translated material, BMJ does not warrant the accuracy and reliability of the translations (including but not limited to local regulations, clinical guidelines, terminology, drug names and drug dosages), and is not responsible for any error and/or omissions arising from translation and adaptation or otherwise.

Open access This is an open access article distributed in accordance with the Creative Commons Attribution Non Commercial (CC BY-NC 4.0) license, which permits others to distribute, remix, adapt, build upon this work non-commercially, and license their derivative works on different terms, provided the original work is properly cited, appropriate credit is given, any changes made indicated, and the use is non-commercial. See: http://creativecommons.org/licenses/by-nc/4.0/.

ORCID iD

Carrie B. Dolan http://orcid.org/0000-0002-4445-7075

\section{REFERENCES}

1 Smith ER, Concepcion TL, Niemeier KJ, et al. Is global pediatric surgery a good investment? World J Surg 2019;43:1450-5.
2 Saxton AT, Poenaru D, Ozgediz D, et al. Economic analysis of children's surgical care in low- and middle-income countries: a systematic review and analysis. PLoS One 2016;11: e0165480.

3 Meara JG, Leather AJM, Hagander L, et al. Global surgery 2030: evidence and solutions for achieving health, welfare, and economic development. The Lancet 2015;386:569-624.

4 Chao TE, Sharma K, Mandigo M, et al. Cost-effectiveness of surgery and its policy implications for global health: a systematic review and analysis. Lancet Glob Health 2014;2:e334-45.

5 Grimes CE, Henry JA, Maraka J, et al. Cost-effectiveness of surgery in low- and middle-income countries: a systematic review. World $\mathrm{J}$ Surg 2014;38:252-63.

6 Qiu X, Nasser JS, Sue GR, et al. Cost-effectiveness analysis of humanitarian hand surgery TRIPS according to WHO-CHOICE thresholds. J Hand Surg Am 2019;44:93-103.

7 Eeson G, Birabwa-Male D, Pennington M, et al. Costs and costeffectiveness of pediatric inguinal hernia repair in Uganda. World $J$ Surg 2015;39:343-9.

8 Poenaru D, Pemberton J, Cameron BH. The burden of waiting: DALYs accrued from delayed access to pediatric surgery in Kenya and Canada. J Pediatr Surg 2015;50:765-70.

9 Hendriks TCC, Botman M, Rahmee CNS, et al. Impact of short-term reconstructive surgical missions: a systematic review. BMJ Glob Health 2019;4:e001176.

10 Smith ER, Concepcion T, Lim S, et al. Disability weights for pediatric surgical procedures: a systematic review and analysis. World J Surg 2018;42:3021-34.

11 Armstrong R, Hall BJ, Doyle J, et al. Cochrane update. 'Scoping the scope' of a cochrane review. J Public Health 2011;33:147-50.

12 Arksey H, O'Malley L. Scoping studies: towards a methodological framework. Int J Soc Res Methodol 2005;8:19-32.

13 Tricco AC, Lillie E, Zarin W, et al. PRISMA extension for scoping reviews (PRISMA-ScR): checklist and explanation. Ann Intern Med 2018;169:467-73.

14 Hughes CD, Babigian A, McCormack S, et al. The clinical and economic impact of a sustained program in global plastic surgery: valuing cleft care in resource-poor settings. Plast Reconstr Surg 2012;130:87e-94.

15 Corlew DS. Estimation of impact of surgical disease through economic modeling of cleft lip and palate care. World J Surg 2010;34:391-6.

16 Alkire B, Hughes CD, Nash K, et al. Potential economic benefit of cleft lip and palate repair in sub-Saharan Africa. World J Surg 2011;35:1194-201.

17 Okafor CE, Onunka O, Idoko LN. Cost-Utility of burns management in Nigeria: a case study of the National orthopaedic Hospital, Enugu. Ann Burns Fire Disasters 2017;30:9.

18 Tadisina KK, Chopra K, Tangredi J, et al. Helping hands: a costeffectiveness study of a humanitarian hand surgery mission. Plast Surg Int 2014;2014:1-12.

19 Shrime MG, Alkire BC, Grimes C, et al. Cost-effectiveness in global surgery: pearls, pitfalls, and a checklist. World J Surg 2017;41:1401-13.

20 Eblovi D, Antúnez M, Clitheroe K, et al. Effectiveness, costeffectiveness, and economic impact of a multi-specialty charitable surgical center in Honduras. International Journal of Surgery Open 2019;20:7-14.

21 Cardarelli M, Vaikunth S, Mills K, et al. Cost-effectiveness of humanitarian pediatric cardiac surgery programs in low- and middle-income countries. JAMA Netw Open 2018;1: e184707.

22 Raj M, Paul M, Sudhakar A, et al. Micro-economic impact of congenital heart surgery: results of a prospective study from a limited-resource setting. PLoS One 2015;10:e0131348.

23 Alkire BC, Shrime MG, Dare AJ, et al. Global economic consequences of selected surgical diseases: a modelling study. Lancet Glob Health 2015;3 Suppl 2:S21-7.

24 Poenaru D, Pemberton J, Frankfurter C, et al. Quantifying the disability from congenital anomalies averted through pediatric surgery: a cross-sectional comparison of a pediatric surgical unit in Kenya and Canada. World J Surg 2015;39:2198-206. 